\title{
Kaposi's sarcoma-associated herpesvirus microRNAs
}

\section{Eva Gottwein *}

Department of Microbiology-Immunology, Feinberg School of Medicine, Northwestern University, Chicago, IL, USA

\section{Edited by:}

Keiji Ueda, Osaka University Graduate

School of Medicine, Japan

\section{Reviewed by:}

Keiji Ueda, Osaka University Graduate

School of Medicine, Japan

Chris Parsons, LSU Health Sciences

Center, USA

\section{*Correspondence}

Eva Gottwein, Department of

Microbiology-Immunology,

Northwestern University, Morton

6-654, 303 East Chicago Avenue,

Chicago, IL 60611, USA.

e-mail: e-gottwein@northwestern.edu
Kaposi's sarcoma-associated herpesvirus (KSHV) is a human pathogenic $\gamma$-herpesvirus strongly associated with the development of Kaposi's Sarcoma and B cell proliferative disorders, including primary effusion lymphoma (PEL). The identification and functional investigation of non-coding RNAs expressed by KSHV is a topic with rapidly emerging importance. KSHV miRNAs derived from 12 stem-loops located in the major latency locus have been the focus of particular attention. Recent studies describing the transcriptomewide identification of mRNA targets of the KSHV miRNAs suggest that these miRNAs have evolved a highly complex network of interactions with the cellular and viral transcriptomes. Relatively few KSHV miRNA targets, however, have been characterized at a functional level. Here, our current understanding of KSHV miRNA expression, targets, and function will be reviewed.

Keywords: KSHV, herpesvirus, microRNA

\section{INTRODUCTION}

Shortly after the discovery of miRNAs as an abundant class of small RNAs in animals (Lee et al., 1993; Pasquinelli et al., 2000; Reinhart et al., 2000; Lagos-Quintana et al., 2001; Lau et al., 2001; Lee and Ambros, 2001), miRNAs of viral origin were identified in EpsteinBarr virus (EBV) infected cells (Pfeffer et al., 2004). Since then, Kaposi's sarcoma-associated herpesvirus (KSHV) and most other herpesviruses were found to encode numerous viral microRNAs (Cai et al., 2005; Pfeffer et al., 2005; Samols et al., 2005; Grundhoff et al., 2006; Grundhoff and Sullivan, 2011; Skalsky and Cullen, 2011).

miRNAs are $\sim 22$ nucleotide (nt) long non-coding RNAs that mediate target mRNA recognition by the miRNA-induced silencing complex (miRISC, referred to as RISC from hereon), which results in mRNA repression. miRNA biogenesis and RISC loading are reviewed in detail elsewhere (Kim et al., 2009) and summarized briefly in Figure 1A. The biogenesis of the large majority of cellular and viral miRNAs, including the KSHV miRNAs, begins with the transcription of capped and polyadenylated primary RNA (pri-miRNA) precursors by RNA polymerase II. miRNA maturation involves two sequential cleavage events. In the nucleus, the endonuclease Drosha excises the $\sim 65 \mathrm{nt}$ pre-miRNA stem-loop, which is exported to the cytoplasm. In the cytoplasm, the premiRNA is further processed by the endonuclease Dicer to produce an imperfect $\sim 20 \mathrm{nt}$ duplex, with $2 \mathrm{nt} 3^{\prime}$ overhangs. The strand with the weaker $5^{\prime}$ base pairing is preferentially incorporated as a mature miRNA into the RISC. The non-incorporated strand, which is unstable and degraded, is termed the star or passenger strand. In cases where mature miRNAs are derived from both arms of the pre-miRNA, these miRNAs are designated $-5 p$ and $-3 p$ to reflect their location in the pre-miRNA.

In the RISC complex, miRNAs are loaded into one of four Argonaute proteins (Agol-4). Imperfect base pairing of the miRNA with sites in mRNAs, most commonly within the $3^{\prime}$ UTR, results in mRNA repression, caused by mRNA destabilization and/or inhibition of translation (Huntzinger and Izaurralde, 2011). Canonical miRNA binding sites minimally exhibit perfect base pairing of the miRNA seed region, i.e. nts $2-7$ from the $5^{\prime}$ end of the miRNA, together with base pairing at nt 8 (7mer-m8site), an adenine (A) across from nt 1 of the miRNA (7mer-Al site), or both (Figure 1B; Bartel, 2009). Less commonly, non-canonical sites with suboptimal seed interactions and $3^{\prime}$ compensatory base pairing or extensive central base pairing confer regulation (Bartel, 2009; Shin et al., 2010). In addition, functional miRNA binding sites in $5^{\prime}$ UTRs and coding sequences (CDS) have also been reported (Grey et al., 2010; Lin and Ganem, 2011). While individual interactions typically have subtle regulatory outcomes, miRNA-mediated regulation is frequently cooperative with multiple sites for the same or different miRNAs in a given mRNA. RISC complexes containing Ago2 can mediate endonucleolytic cleavage of target mRNAs upon perfect base pairing between the entire miRNA sequence and its target (Hutvagner and Zamore, 2002). While this property of Ago2 is commonly exploited for miRNA activity assays, recognition of perfect targets by mammalian miRNAs occurs only rarely. Several hundred conserved human miRNAs have been identified and each miRNA can target hundreds of mRNAs, suggesting that most mRNAs and biological pathways are subject to miRNA-mediated regulation.

The human $\gamma$-herpesvirus KSHV is the etiological agent of Kaposi's Sarcoma, a complex neoplasm driven by KSHV-infected endothelial cells. KSHV is also strongly associated with the B cell proliferative disorder primary effusion lymphoma (PEL) and some cases of multicentric Castleman's disease (MCD). Like all herpesviruses, KSHV can enter a latency phase with highly restricted protein expression, thus limiting immune exposure while allowing persistence of the virus. During latency, KSHV expresses viral miRNAs from 12 pre-miRNAs in addition to a handful of latent proteins (Cai et al., 2005; Pfeffer et al., 2005; Samols et al., 2005; Grundhoff et al., 2006). Possible advantages of using miRNAmediated regulation for herpesviruses may be that miRNAs are not 


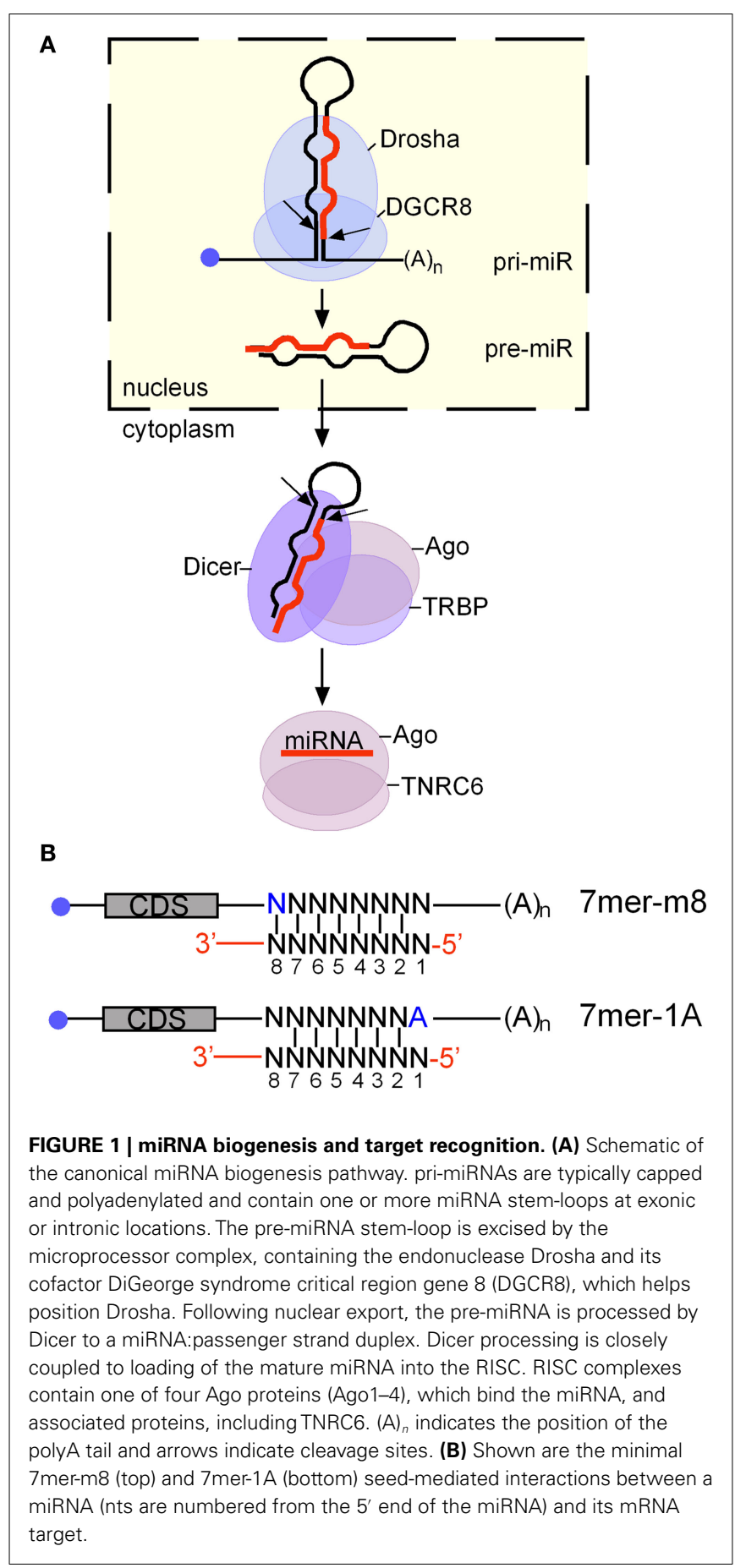

antigenic and their precursors can easily fit into multifunctional transcripts (Cullen, 2006). In addition, the regulatory potential of miRNAs differs fundamentally from that of proteins, i.e., they are commonly multifunctional and may allow viruses to regulate combinations of mRNAs and pathways that would be hard to access otherwise. Here, I review what is currently known about KSHV miRNA expression, targets and functions. miRNAs encoded by other viruses are reviewed elsewhere (Cullen, 2011; Grundhoff and Sullivan, 2011).

\section{THE KSHV miRNAs}

\section{LOCUS}

Kaposi's sarcoma-associated herpesvirus produces mature miRNAs from 12 stem-loops, all of which are encoded in the latency locus (Figure 2A). The latency region is complex and allows for the coordinated expression of the KSHV miRNAs with the viral proteins latency-associated nuclear antigen (LANA), FADD-like interleukin-1- $\beta$-converting enzyme (FLICE)/caspase8-inhibitory protein (v-FLIP), v-cyclin, and Kaposins A-C (Figure 2A). The viral latent proteins mediate the episomal maintenance of the KSHV genome (LANA), stimulate NFKB signaling (v-FLIP), and modulate cell cycle progression ( $\mathrm{v}$-cyclin), among other functions. The mature KSHV miRNAs are called miR-K121 to miR-K12-12, based on their proximity to the kaposin (K12) gene, or simply miR-K1 to miR-K12. The transcripts from the latency region that serve as pri-miRNAs for the KSHV miRNAs are shown in Figure 2B (Cai and Cullen, 2006). All of these transcripts presumably also function as mRNAs for one or more of the Kaposin proteins, whose coding sequences are located in the common $3^{\prime}$ portion of these transcripts. The promoters with start sites at 127880/86 and 123751/60 are active during latency and the resulting pri-miRNAs contain miR-K1-K9 and miR-K11 within a $\sim 4.8-\mathrm{kb}$ intron and miR-K10/miR-K12 in their $3^{\prime}$ terminal exon. At least in the PEL cell line BC-1, a singly spliced mRNA with a $29-\mathrm{kb}$ intron containing miR-K1-K9 and miRK11 also exists (Figure 2B, bottom). The latency promoters also drive the expression of mRNAs encoding LANA, $\mathrm{v}$-cyclin, and $\mathrm{v}$ FLIP (not shown in Figure 2B), which terminate downstream of the v-FLIP coding region and do not contain the miRNA hairpins (Cai and Cullen, 2006). In addition, there is a predominantly lytic promoter which gives rise to an unspliced $\sim 1.3 \mathrm{~kb}$ transcript that was shown to minimally express Kaposin B and also contains the miR-K10 and miR-K12 stem-loops, resulting in increased levels of miR-K10 and miR-K12 expression during lytic replication.

Virtually all of the KSHV pre-miRNAs overlap multifunctional regions. The entire miR-K10 stem-loop is embedded within the open reading frame of the Kaposin A and C proteins at a location that is also in the $3^{\prime}$ UTR of Kaposin B and the miR-K12 stem-loop is located in the $3^{\prime}$ UTRs of all Kaposin transcripts. Because the processing of these two exonic miRNAs and Kaposin translation are mutually exclusive events, the miR-K10 and miR-K12 stemloops can be viewed as negative cis-regulatory RNA elements that mediate the nuclear destabilization of Kaposin mRNAs and consequently reduce protein expression from these transcripts (Lin and Sullivan, 2011). The miR-K9 stem-loop is located within a $\sim 1.7-\mathrm{kb}$ sequence that can function as an origin of lytic genome replication (ORI-lytB; AuCoin et al., 2002; Lin et al., 2003). Much of this sequence represents an inverted repeat of a second origin of lytic replication (Ori-lytA), between ORF K4.2 and K5. In contrast to ORI-lytA, which is essential for replication, ORI-lytB is not required for genome replication and the function of this sequence in the context of the virus remains unknown (Xu et al., 2006). The miR-K1-K7 and miR-K11 stem loops are antisense to the recently identified $10 \mathrm{~kb}$ antisense to latency transcripts (ALT) RNA (Figure 2A; Chandriani et al., 2010). ALT is a non-coding RNA of unknown function expressed during lytic replication. 


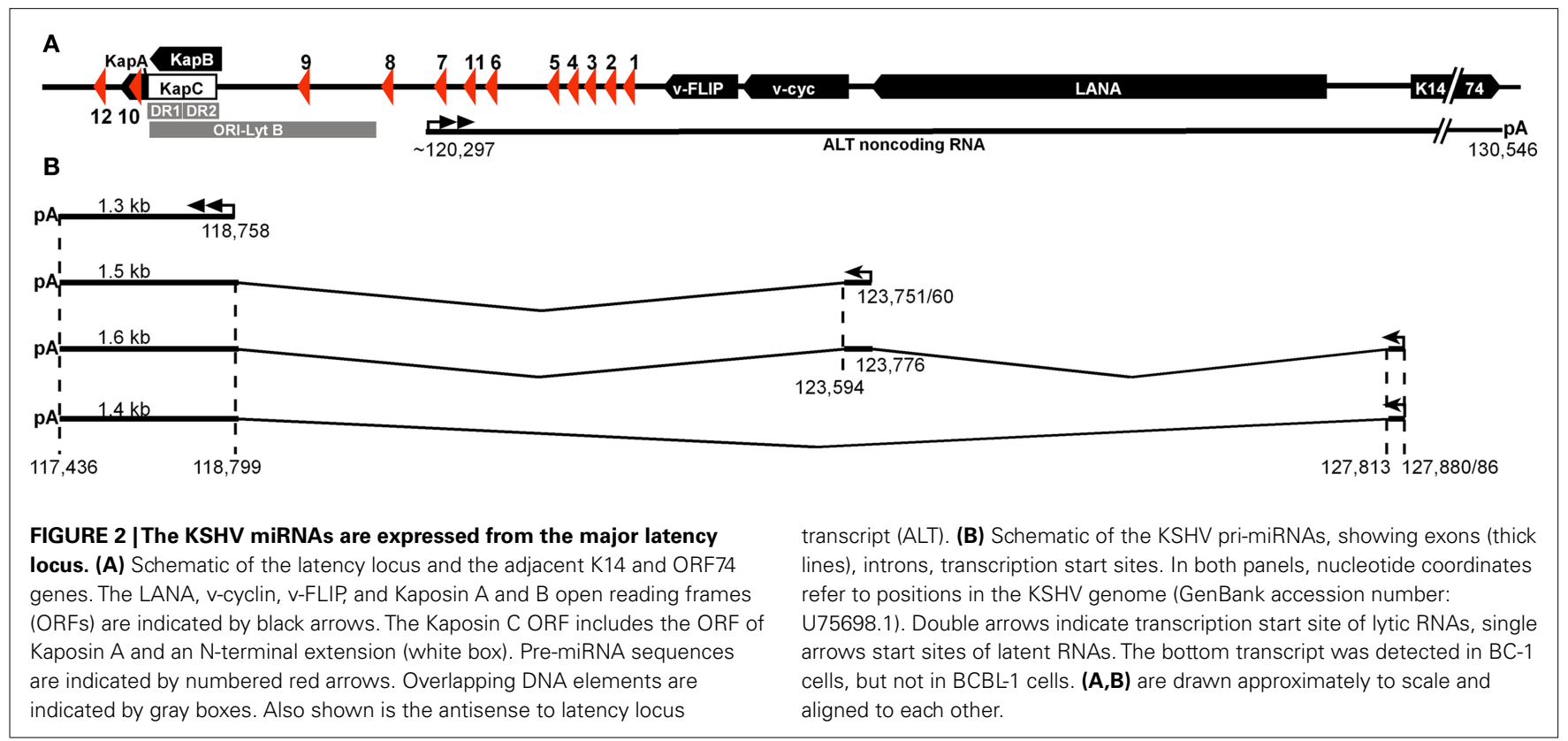

While the ALT promoter has not been mapped, it may well overlap the miR-K8 stem-loop, which begins only 300 nts away from the ALT start site. Finally, the expression of sequences antisense to the miR-K8 stem-loop has been reported, although an antisense transcript in this region has not been identified (Lin et al., 2010; Umbach and Cullen, 2010).

\section{SEQUENCES OF THE KSHV miRNAs}

The KSHV miRNAs were first identified by cDNA cloning and traditional sequencing (Cai et al., 2005; Pfeffer et al., 2005; Samols et al., 2005) and by an approach that combined the computational prediction of candidate pre-miRNA stem-loops with highthroughput validation of miRNA expression (Grundhoff et al., 2006). These findings were further extended by small RNA deepsequencing on the Illumina and ABI SOLiD platforms (Lin et al., 2010; Umbach and Cullen, 2010; Gottwein et al., 2011). Table 1 lists the sequences of the mature KSHV miRNAs that are likely to be expressed at physiologically relevant levels under most conditions. This list is based on the number of reads obtained in deep-sequencing experiments, miRNA activity assays and detection of the miRNAs using qRT-PCR, primer extension analysis, or northern blotting. The actual number of KSHV miRNAs exceeds that of the 12 stem-loop precursors, due to the expression of relatively abundant $5 p$ and $3 p$ miRNAs from several of the premiRNAs and other sources of miRNA sequence variation discussed below. While $5 \mathrm{p}$ and $3 \mathrm{p}$ sequences from all stem-loop precursors have been detected at high sequencing depth (Lin et al., 2010; Umbach and Cullen, 2010), it remains to be seen if all of these sequences are expressed at functionally significant levels in every context. Expression of $5 p$ and $3 p$ miRNAs was consistently observed for some KSHV miRNAs (i.e., miR-K4, miR-K6, miR$\mathrm{K} 7$, miR-K8, and miR-K9). In contrast, other sequences that were recovered by deep-sequencing (e.g., miR-K1-3p and miR-K3-3p) probably reflect true star strands without functional relevance in most settings. The KSHV miRNA repertoire is further expanded by differential processing at the $5^{\prime}$ end of miR-K10, which results in two distinct seed sequences for this miRNA (Umbach and Cullen, 2010), and through adenosine (A) to inosine (I) editing by RNA-specific adenosine deaminase (ADAR) of each of the miR-K10 seeds. Unedited and A to I edited miR-K10 are designated miR-K10a and miR-K10b, respectively (Table 1; Pfeffer et al., 2005). The longer $5^{\prime}$ processing variants of miR-K10 are designated miR-K10 + 1_5 (Table 1; Gottwein et al., 2011). During latency, expression of miR-K10b is low, but RNA editing at this location is induced during lytic replication and it is possible that a substantial fraction of miR-K10 expressed during lytic reactivation has undergone A to I editing (Gandy et al., 2007). Because I base-pairs like guanosine $(\mathrm{G}), \mathrm{miR}-\mathrm{K} 10 \mathrm{~b}$ is expected to target a different pool of mRNAs than miR-K10a. An interesting case is miR-K3, with several PEL cell lines encoding a novel miR-K3 sequence with an additional $5^{\prime}$ adenosine (miR-K3 + 1_5; Gottwein et al., 2011). As in the case of miR-K10, acquisition of the additional miRNA seed sequence is expected to extend the target pool of miR-K3.

\section{CELLULAR ANALOGS OF THE KSHV miRNAs}

Importantly, several of the KSHV miRNAs share seed homology with cellular miRNAs suggesting that they function as viral analogs of these miRNAs (Figure 3A). Such functional analogy has been demonstrated for miR-K11, which mimics miR-155 (Gottwein et al., 2007; Skalsky et al., 2007), and for the two miR-K10a miRNAs, which together mimic one of two miR-142-3p derived miRNAs (Gottwein et al., 2011). Interestingly, miR-K3+1_5 shares 7 mer seed homology with the miR-23 miRNA family, but functional analogy between these miRNAs has not been demonstrated yet. A final analogy with likely functional significance is the 6mer seed homology between miR-K6-5p and the miR-214 family of miRNAs. Interestingly, miR-K6-5p also shares extended homology with the miR-15/miR-16 family of miRNAs (Skalsky et al., 2007), but the seed is offset by $1 \mathrm{nt}$ (Figure 3A). For 6mer 
Table 1 | Sequences of the KSHV miRNAs.

\begin{tabular}{lll}
\hline miRNA & Arm & Sequence \\
\hline miR-K1-5p & $5 p$ & AUUACAGGAAACUGGGUGUAAG(CUG) \\
miR-K2-5p & $5 p$ & AACUGUAGUCCGGGUCGAU(CUGA) \\
miR-K3-5p & $5 p$ & UCACAUUCUGAGGACGGCAGCGA(CG) \\
miR-K3_+1_5 & $5 p$ & AUCACAUUCUGAGGACGGCAGCGA \\
miR-K4-3p & $3 p$ & UAGAAUACUGAGGCCUAGCUG(A) \\
miR-K4-5p & $5 p$ & AGCUAAACCGCAGUACUCUAGG \\
miR-K5-3p & $3 p$ & UAGGAUGCCUGGAACUUGCCGG(U) \\
miR-K6-3p & $3 p$ & UGAUGGUUUUCGGGCUGUUGAG(C) \\
miR-K6-5p & $5 p$ & CCAGCAGCACCUAAUCCAUCGG \\
miR-K7-3p & $3 p$ & UGAUCCCAUGUUGCUGGCGC(UCA) \\
miR-K7-5p & $5 p$ & AGCGCCACCGGACGGGGAUUUAUG \\
miR-K8-3p & $3 p$ & CUAGGCGCGACUGAGAGAGC(AC) \\
miR-K8-5p & $5 p$ & ACUCCCUCACUAACGCCCCGCU \\
miR-K9-3p & $3 p$ & CUGGGUAUACGCAGCUGCGU(AA) \\
miR-K9-5p & $5 p$ & ACCCAGCUGCGUAAACCCCG(CU) \\
miR-K10a $(-3 p)^{c}$ & $3 p$ & UAGUGUUGUCCCCCCGAGUGG(C) \\
miR-K10b- $(-3 p)^{b, c}$ & $3 p$ & UIGUGUUGUCCCCCCGAGUGG(C) \\
miR-K10a_+1_5 (-3p) & $3 p$ & UUAGUGUUGUCCCCCCGAGUGG(C) \\
miR-K10b_+1_5 (-3p) $)^{b, c}$ & $3 p$ & UUIGUGUUGUCCCCCCGAGUG(C) \\
miR-K11-3p & $3 p$ & UUAAUGCUUAGCCUGUGUCCG(AU) \\
miR-K12-3pc & $3 p$ & UGGGGGAGGGUGCCCUGGUUG(A) \\
miR-K12-5pc & $5 p$ & AACCAGGCCACCAUUCCUCUCCG \\
\hline S & & \\
\hline
\end{tabular}

Sequences of the KSHV miRNAs with significant expression under most experimental conditions are listed. Nucleotides in brackets are not always present in deep-sequencing reads. Bold letters highlight the A to I editing event within miR-K10. ${ }^{a}$ miR-K3+1_5 is only present in a subset of KSHV strains. ${ }^{b}$ miR-K10b may reach functionally relevant levels of expression only during lytic replication. ${ }^{c}$ miR-K10 and miR-K12 expression is increased during lytic replication. In the case of miR-K10, it is unclear if the expression of unedited and/or edited miRNAs is increased.

seed and offset homology, resulting functional analogies would be expected to be less complete than for $7 \mathrm{mer}$ seed analogs. Other potential analogs have been listed (Gottwein et al., 2011), but in most cases the cellular miRNAs in question have not undergone stringent validation. Findings in mice suggest that many of the small RNAs currently annotated as miRNAs may not represent true miRNAs (Chiang et al., 2010). It is therefore possible that many of the $>1000$ human miRNAs listed in miRBase have been misclassified as miRNAs. The significance of analogies with such uncharacterized miRNAs is therefore unclear and could be co-incidental (Grundhoff and Sullivan, 2011).

\section{BIOLOGICAL SIGNIFICANCE OF KSHV miRNA EXPRESSION LEVELS}

The regulatory impact of miRNA expression depends on the relative abundance of the miRNA and its targets. Consequently, the level of miRNA expression is of critical relevance for miRNA function. Methods used for miRNA detection have intrinsic biases and a careful analysis of the absolute and relative expression levels of individual KSHV miRNAs against oligonucleotide standards has not been reported. However, from deep-sequencing data, real-time PCR experiments and activity assays, it appears that individual KSHV miRNAs are expressed at dramatically different copy

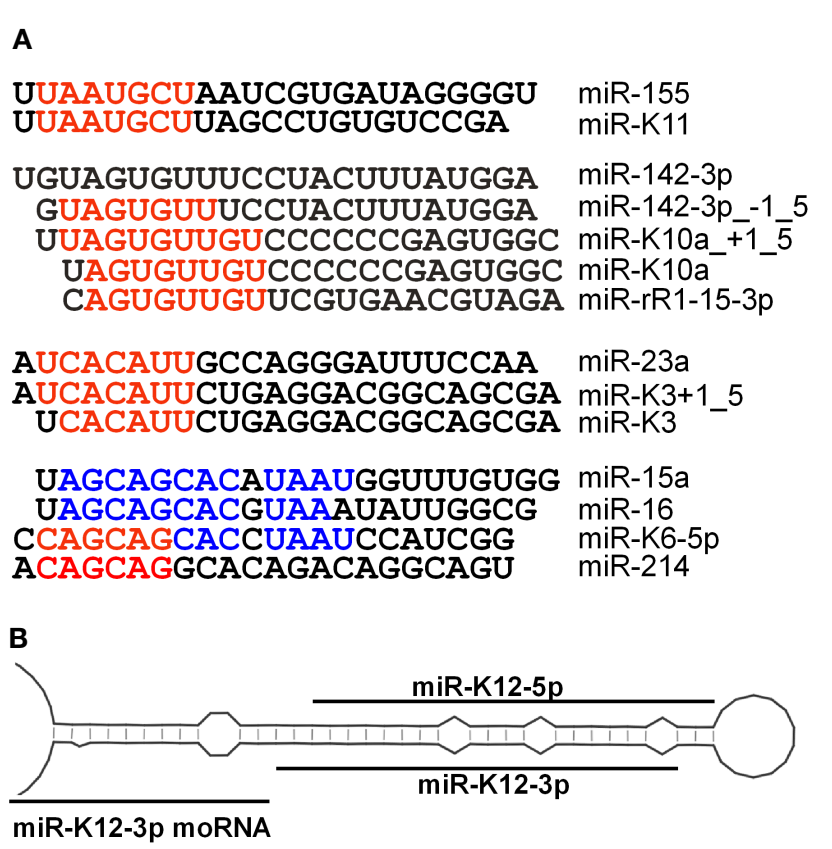

FIGURE 3 | (A) KSHV miRNAs with known or potential cellular analogs. miRNAs are aligned to show seed (red) and non-seed (blue) homologies. (B) Schematic illustrating the origin of moRNAs.

numbers. While some KSHV miRNAs are always readily detectable (especially miR-K1, miR-K3, miR-K4-3p, miR-K6-3p, miR-K11), others are unlikely to be abundant (e.g., miR-K9; Cai et al., 2005; Pfeffer et al., 2005; Gottwein et al., 2006, 2011; O'Hara et al., 2009; Hansen et al., 2010; Lin et al., 2010; Umbach and Cullen, 2010). Because all KSHV miRNAs are expressed from common pri-miRNAs, their differential expression must result from differences in processing efficiency, RISC loading, and/or stability. In addition, the expression level of the KSHV miRNAs also varies among PEL cell lines and de novo infection models (Hansen et al., 2010; Gottwein et al., 2011). While some PEL cell lines express relatively low levels of the KSHV miRNAs ( 20-30\% of all miRNA reads in deep-sequencing experiments, e.g., BC-1 and BCBL-1), other PEL cell lines express very high levels of the KSHV miRNAs ( $>80 \%$ of all miRNA reads, BC-3 cells). The KSHV miRNAs are constitutively expressed during latency and their expression is maintained upon entry into lytic replication (Cai et al., 2005; Pfeffer et al., 2005). miR-K10 and miR-K12 are induced several-fold during lytic replication due to their expression from both latent and lytic transcripts (see above). In addition, RNA editing of the miR-K10 stem-loop is likely induced during lytic replication, but it is unclear if this results in increased expression of miR-K10b over miR-K10a (Gandy et al., 2007). Finally, the expression levels of the KSHV miRNAs during natural KSHV infection remain unknown.

\section{EVOLUTIONARY CONSERVATION AND VARIATION BETWEEN KSHV STRAINS}

Like most herpesvirus miRNAs (Cai et al., 2006; Walz et al., 2010), the KSHV miRNA seed sequences are not conserved between 
KSHV and evolutionary distant herpesviruses. One notable exception is the 6mer seed homology between KSHV miR-K10a and miR-rR1-15-3p of the closely related rhesus rhadinovirus (RRV, Figure 3A; Umbach and Cullen, 2010), which may be the result of convergent evolution rather than true evolutionary conservation. Despite this lack of evolutionary conservation of the miRNA seeds between evolutionary distant viruses, the mature sequences of the KSHV miRNAs appear to be highly conserved between KSHV isolates (Marshall et al., 2007, 2010). Maybe surprisingly, Marshall et al. also reported a high degree of conservation of sequences outside the mature miRNAs. For example the terminal loop sequences of the pre-miRNA stem-loops were generally conserved, despite the fact that these sequences are expected to be functionally irrelevant as long as the stem-loop structure is maintained. Because of the multifunctional nature of the latency region, it is possible that yet unappreciated selective pressures act on these sequences. Despite this caveat, the observed degree of miRNA conservation strongly argues that the KSHV miRNAs are important for KSHV. Several polymorphisms that change the KSHV miRNA repertoire have been described. An A to G polymorphism in the miR-K5 passenger strand (position 121,315) was found in $>20 \%$ of all KSHV sequences analyzed (Marshall et al., 2007, 2010). This single nucleotide polymorphism (SNP) was reported elsewhere to alter the structure of the miR-K5 stem, which results in reduced processing of pri-miR-K5 by Drosha and lower levels of miR-K5 expression (Cai et al., 2005; Gottwein et al., 2006, 2011). The miRK9 stem-loop appears to be the most variable between isolates and has been lost from at least one PEL cell line (BC-3; Marshall et al., 2007; Umbach and Cullen, 2010), suggesting that this miRNA is dispensable for the maintenance of latency, for lytic reactivation and possibly for lymphomagenesis by KSHV. Other reported variants of pre-miR-K9 are likely to also dramatically alter either miR-K9 sequence or expression (Marshall et al., 2007, 2010). Other frequent polymorphisms are located outside mature or passenger strand sequences and consequences on miRNA expression have not been reported (Marshall et al., 2007, 2010).

\section{OTHER SMALL RNAs EXPRESSED FROM THE KSHV LATENCY REGION}

Two reports described the detection of miRNA offset RNAs (moRNAs or moRs) and small RNAs antisense to the KSHV miRNAs (Lin et al., 2010; Umbach and Cullen, 2010). moRNAs were first described in the sea squirt Ciona intestinalis, which serves as a model organism for simple chordates (Shi et al., 2009). moRNAs are processed from sequences adjacent to the pre-miRNA stem-loop (Figure 3B) and are both frequent and abundant in $C$. intestinalis. moRs have also been detected in human small RNA deep-sequencing libraries, but at levels consistently below that of miRNAs and passenger strand sequences (Langenberger et al., 2009), suggesting that these RNAs are potentially only byproducts of miRNA biogenesis. KSHV moRNAs were detected for 9 of $12 \mathrm{KSHV}$ miRNA stem-loops (Lin et al., 2010; Umbach and Cullen, 2010), but like human moRs, these sequences were of very low abundance. While RRV moRs have shown activity in cleavage assays for miRNA activity when expressed at high enough levels (Umbach et al., 2010), the low expression of KSHV moRs during KSHV infection suggests that these sequences might not be expressed at high enough levels to be functionally relevant.
Similarly, small RNAs antisense to miRNA hairpins for miR-K2, miR-K4, and miR-K8 were recovered at frequencies lower than for passenger strand miRNAs (Lin et al., 2010; Umbach and Cullen, 2010). These sequences arise due to bi-directional transcription of a miRNA locus. The expression of KSHV antisense miRNAs appears to be restricted to lytic replication and the recovery of $-5 p$ and $-3 p$ sequences suggests that they are products of the miRNA biogenesis machinery. A small RNA antisense to miR-K4 (miRK4-AS-3p) was readily detectable and active in miRNA indicator assays when this region was placed into an expression vector (Lin et al., 2010). It is unclear, however, if this is also the case at physiological levels of expression in PEL. miR-K4-AS and miR-K2-AS could be derived from the ALT RNA. No transcript encompassing miR-K8-AS sequences has been identified to date.

\section{TARGETS AND FUNCTIONS OF THE KSHV miRNAs}

The constitutive expression and conservation of the KSHV miRNAs suggests they are functionally important during natural KSHV infection and may also contribute to the oncogenic properties of KSHV. Thus, identifying the targets and functions of the KSHV miRNAs is clearly important for our understanding of KSHV biology and pathogenesis. In principle, the KSHV miRNAs may have evolved to target cellular or viral mRNAs and to regulate cellular properties or viral gene expression. As discussed above, KSHV has evolved a number of novel miRNA seed sequences and also appears to access pre-existing, evolutionary conserved regulatory networks by encoding miRNAs with seed homology to evolutionary conserved cellular miRNAs. While cellular miRNAs are thought to repress many mRNAs, perhaps even hundreds, through seed interactions, there is no good reason to assume that novel viral miRNAs would also target multiple imperfect sites and predominantly engage in seed-mediated interactions. However, no cleavage targets of the KSHV miRNAs have been identified to date and it appears all KSHV miRNAs have many seed targets much like typical cellular miRNAs. It is noteworthy that the KSHV miRNAs must have undergone selection to be functionally compatible with all cell types relevant to KSHV infection, including endothelial cells and $\mathrm{B}$ cells, each with a different transcriptome. Consequently, it is possible that one or more targets with a strong selective advantage in one cell type or the latent or lytic stages of KSHV infection may come at a cost in other cell types or at other stages of the life cycle.

\section{KSHV MIRNA TARGETOME}

Understanding the KSHV miRNA targetome is a critical step toward identifying the functions of the KSHV miRNAs. One of the most successful methods for the identification of functionally relevant targets of evolutionary conserved cellular miRNAs is the computational identification of evolutionary conserved seed matches in 3' UTR sequences (Bartel, 2009). The assumption underlying this approach is that miRNA and target sequences co-evolve and that functionally important interactions will be conserved. With the exception of the viral analogs of cellular miRNAs, the KSHV miRNAs are unique to KSHV, and therefore not expected to preferentially target $3^{\prime}$ UTR sequences that are evolutionary conserved between species. Consequently, computational target prediction is generally not suitable for the identification of targets of the KSHV miRNAs with novel seeds, which 
has mostly relied on experimental target identification. Several studies combined gene expression profiling upon ectopic delivery of individual or several KSHV miRNAs (Gottwein et al., 2007; Samols et al., 2007; Skalsky et al., 2007; Ziegelbauer et al., 2009; Hansen et al., 2010; Suffert et al., 2011) or their antagonism in the context of KSHV infection (Ziegelbauer et al., 2009) with the detection of seed matches to the expressed miRNA(s) among differentially expressed mRNAs in order to identify candidate targets. While such analyses can also give insight into the functional consequences of miRNA expression, the identification of direct targets from differentially expressed mRNAs is confounded by indirect consequences of miRNA expression. In addition, while miRNAinduced changes of mRNA expression are often predictive of the effect on protein expression (Baek et al., 2008; Selbach et al., 2008), the effect of individual interactions is typically small and many targets may be missed using this approach. To overcome these limitations, Dölken et al. (2010) used Ago2-immunoprecipitation coupled with microarray analysis of associated RNAs (RIP-Chip) to identify mRNAs that are enriched in RISC complexes in cells expressing KSHV miRNAs compared to control cells. This work resulted in a list of 114 high confidence candidate targets of the KSHV miRNAs. While RIP-Chip can measure the degree of mRNA association with RISC, it cannot confidently assign the targeting miRNA or map its binding site. This caveat of RIP-CHIP is overcome by the transcriptome-wide identification of miRNA binding sites using photoactivatable-ribonucleoside-enhanced crosslinking and immunoprecipitation (PAR-CLIP) and high-throughput sequencing of RISC protected fragments (Hafner et al., 2010; Gottwein et al., 2011). In contrast to other technologies, PAR-CLIP directly identifies RISC-binding sites, which are examined for miRNA seed matches. Applying PAR-CLIP to PEL cell lines has yielded thousands of experimentally identified Ago2 binding sites with seed matches to KSHV miRNAs, in more than 2000 candidate target mRNAs. Validation of a subset of these candidate targets by reporter assays suggested that $\geq 75 \%$ of the identified sites can be expected to cause measurable regulation by the assigned miRNA. Thus, extensive lists of candidate targets of the KSHV miRNAs and their likely binding sites exist, but further validation of individual interactions will be required. Because most of these experiments were performed in B cells and none of the datasets is expected to have captured all interactions of the KSHV miRNAs, future work will have to refine our understanding of the KSHV miRNA targetome in other cell types and to address which of the identified interactions impact gene expression and are functionally relevant. Despite these caveats, pathway analyses of high confidence candidates can already give insight into potential functions of the KSHV miRNAs (Dölken et al., 2010; Gottwein et al., 2011). While such analyses are still hampered by the incomplete functional annotation of the human transcriptome and may be biased by the cell type candidates were derived from, current data strongly suggest that KSHV miRNAs are highly multifunctional. Candidate targets of the KSHV miRNAs were found to be significantly enriched for those with roles as transcription factors and those that participate in signaling and vesicular trafficking, among others. The notion that the KSHV miRNAs have diverse functions is also supported by functional validation of diverse targets (below). PAR-CLIP and/or candidate approaches, have also resulted in a number of known or suspected viral targets of the KSHV miRNAs, including the mRNA encoding replication and transcription activator (RTA; Bellare and Ganem, 2009; Gottwein et al., 2011). Finally, a surprisingly high overlap between PAR-CLIP targets of the KSHV and EBV miRNAs, suggests that the KSHV and EBV miRNAs share many functions as a result of convergent evolution (Gottwein et al., 2011). While this cannot be said for sure until such common targets and functions are studied in detail, this hypothesis is already supported by the finding that KSHV, human cytomegalovirus (HCMV) and EBV all target $\mathrm{MICB}$, an important mediator of $\mathrm{NK}$ cell recognition, through different sites (Nachmani et al., 2009). The overlapping target range of EBV and KSHV miRNAs may have functional significance in PEL, because $>80 \%$ of PELs carry both EBV and KSHV (Cesarman et al., 1995) and co-express > 30 EBV BART miRNAs with the KSHV miRNAs (Cai et al., 2006; Gottwein et al., 2011). It is therefore likely that the KSHV and EBV miRNAs synergize in PEL to repress important targets. Indeed, PAR-CLIP data predicts that several of the already validated KSHV miRNA targets may also be targeted by EBV miRNAs, including for example caspase 3 (Suffert et al., 2011). In summary, while a significant amount of work remains, we are beginning to understand the complexity of the KSHV miRNA targetome. An even higher degree of complexity is certain to emerge once additional cell types are analyzed at comparable depth.

The functional validation of miRNA targets typically includes several steps: (1) The $3^{\prime}$ UTR sequence of the candidate target is placed $3^{\prime}$ to a luciferase reporter and the specific regulation of indicator activity by the targeting miRNA is established by $3^{\prime}$ UTR reporter assays. The loss of reporter regulation upon mutation of the miRNA binding site is currently the only way to demonstrate the potential of a direct regulatory interaction in human cells. However, due to the artificiality of this assay, target regulation in a $3^{\prime}$ UTR reporter assay cannot prove that the interaction does indeed take place in the context of infection or is functionally relevant. (2) Repression of the endogenous protein by the miRNA is established. This is demonstrated by reduced target expression following the ectopic expression of physiological levels of the targeting miRNA in cells lacking this miRNA and by increased target expression following the inhibition of the miRNA in the context of infection. Because miRNA-induced changes are often subtle, quantitative western blotting is emerging as one of the best tools to accurately establish the level of regulation at the protein level (Abend et al., 2010). (3) The contribution of target regulation to observed functional consequences is established. Because it is often hard to unequivocally demonstrate that observed functional consequences are indeed due to the targeting of a particular mRNA, several lines of indirect evidence are usually accumulated to support this idea. Upon ectopic miRNA expression, rescue of a miRNA-induced phenotype can be performed by re-expression of the target. In addition, RNA interference (RNAi) of the target should phenocopy miRNA expression. Conversely, upon miRNA inhibition, siRNA of the target mRNA is expected to reverse the phenotype and overexpression of the target is expected to phenocopy miRNA knockdown. The few KSHV miRNA targets that have undergone fairly stringent functional validation are listed in Table 2. In the following paragraphs, key concepts and open questions that have emerged are highlighted. 
Table 2 | KSHV miRNA targets.

\begin{tabular}{|c|c|c|c|}
\hline Gene Symbol & miRNA(s) & Reference & Functional consequences \\
\hline $\mathrm{BACH} 1$ & miR-K11 (PC) & $\begin{array}{l}\text { Gottwein et al. (2007), Skalsky et al. (2007), } \\
\text { Qin et al. (2010a) }\end{array}$ & $\begin{array}{l}\text { Upregulation of SCL7A11/xCT expression by the KSHV } \\
\text { miRNAs, increased viability under oxidative stress }\end{array}$ \\
\hline BCLAF1 & $\begin{array}{l}\text { K5 } \\
\text { K9-3p } \\
\text { K10a (PC), K10b }\end{array}$ & Ziegelbauer et al. (2009) & Prime cells for $\mathrm{KSHV}$ reactivation, modulate caspase activity \\
\hline CASP3 & $\begin{array}{l}\operatorname{miR}-\mathrm{K} 1(\mathrm{PC}) \\
\operatorname{miR}-\mathrm{K} 3(\mathrm{PC}) \\
\operatorname{miR}-\mathrm{K} 4-3 \mathrm{p}(\mathrm{PC})\end{array}$ & Suffert et al. (2011) & $\begin{array}{l}\text { This interaction may contribute to the inhibition of apoptosis } \\
\text { by KSHV }\end{array}$ \\
\hline CDKN1A/p21 & miR-K1 (PC) & Gottwein and Cullen (2010) & Attenuation of p21-dependent cell cycle arrest \\
\hline $\mathrm{IKBKE} / \mathrm{IKK} \varepsilon$ & miR-K11 & Gottwein et al. (2007), Liang et al. (2011) & $\begin{array}{l}\text { Attenuation of interferon induction by pattern recognition } \\
\text { receptors, stabilize KSHV latency }\end{array}$ \\
\hline MAF & $\begin{array}{l}\text { miR-K1 } \\
\text { miR-K6-5p } \\
\text { miR-K11 }\end{array}$ & Hansen et al. (2010) & Downregulation of LEC marker expression, de-differentiation \\
\hline MICB & miR-K7 & Nachmani et al. (2009) & Reduced NK cell recognition and activation \\
\hline NFIB & miR-K3 & Lu et al. (2010a) & $\begin{array}{l}\text { Stabilization of KSHV latency by regulation of RTA promoter } \\
\text { activity }\end{array}$ \\
\hline $\mathrm{NFKBIA} / I_{\kappa} \mathrm{B} \alpha$ & miR-K1 & Lei et al. (2010) & $\begin{array}{l}\text { Stabilization of KSHV latency by regulation of RTA promoter } \\
\text { activity }\end{array}$ \\
\hline $\mathrm{RBL} 2$ & miR-K4-5p (PC) & Lu et al. (2010b) & $\begin{array}{l}\text { De-repression of DNA methyl transferases Dnmt1, 3a } \\
\text { and 3b? }\end{array}$ \\
\hline SMAD5 & miR-K11 (PC) & Liu et al. (2012) & Attenuation of TGF-beta signaling \\
\hline TNFRSF12A/TWEAKR & miR-K10a (PC) & Abend et al. (2010) & $\begin{array}{l}\text { Reduce cellular responses to TWEAK signaling, including } \\
\text { the induction of inflammatory cytokines and the induction of } \\
\text { apoptosis }\end{array}$ \\
\hline KSHV RTA (ORF50) & miR-K9-5p & Bellare and Ganem (2009) & $\begin{array}{l}\text { Reduce RTA expression and consequently inhibit KSHV } \\
\text { reactivation }\end{array}$ \\
\hline
\end{tabular}

Only KSHV miRNA targets that have undergone stringent validation and at least some functional analyses are listed. The gene symbol, the targeting miRNA(s), original

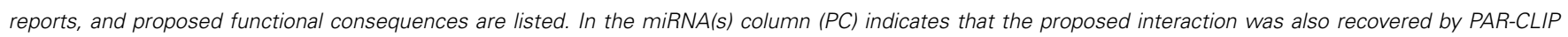
(Gottwein et al., 2011).

\section{KSHV ANALOGS OF CELLULAR miRNAs}

Kaposi's sarcoma-associated herpesvirus gains access to a number of pre-existing regulatory networks by encoding viral analogs of cellular miRNAs. As mentioned above, miR-K11 shares $7 \mathrm{mer}$ seed homology with miR-155 and their overlapping target range has been extensively validated: ectopically expressed miR-K11 and miR-155 regulate similar sets of mRNAs and result in equivalent regulation of $3^{\prime}$ UTR reporters in all cases tested to date (Gottwein et al., 2007; Skalsky et al., 2007). Similar target regulation by miR-K11 and miR-155 was also observed at the level of protein expression for the transcription factors $\mathrm{BACH} 1$ and FOS in side-by-side experiments. In addition, it was demonstrated that miR-K11 regulates protein expression of known miR-155

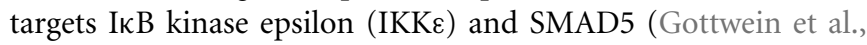
2007; Lu et al., 2008; Yin et al., 2008, 2010; Liang et al., 2011; Liu et al., 2012). More recently, the analogy between miR-K11 and miR-155 was further confirmed by the recovery of $\sim 40 \%$ of all known miR-155 targets as candidate targets of miR-K11 in PEL cells using PAR-CLIP (Gottwein et al., 2011). While a role of miR-K11 in KSHV-induced cancer has not been demonstrated, such a role appears likely, because (1) the constitutive expression of its analog miR-155 in B cells or hematopoietic stem cells causes B cell- or myelo-proliferative disease (Costinean et al., 2006; O'Connell et al., 2008), (2) EBV-induced cellular miR-155 is essential for the survival and growth of EBV-transformed lymphoblastoid cells (Linnstaedt et al., 2010), (3) a viral miR-155 analog encoded by the oncogenic chicken $\alpha$-herpesvirus Marek's disease virus (MDV) was found to be essential for lymphomagenesis by MDV in chickens (Zhao et al., 2011), and (4) the constitutive expression of miR-K11 in human hematopoietic cells that were reconstituted into a humanized mouse model phenocopied expression of miR-155 and lead to an expansion of splenic B cells (Boss et al., 2011). Which of the many miR- 155 targets are responsible for these observations is not understood in detail, but it is tempting to speculate that at least some of the relevant targets may be conserved in humans, mice, and chicken. In addition to a potential role in lymphomagenesis, miR-K11 contributes to the KSHV-induced transcriptional reprogramming of lymphatic endothelial cells (Hansen et al., 2010), promotes cell survival under oxidative stress (Qin et al., 2010a), and inhibits TGF-beta signaling (Liu et al., 2012). These functions of miR-K11 are discussed below. 
Recently, a more complex analogy has been demonstrated between KSHV miR-K10a and the cellular miRNA miR-142-3p (Gottwein et al., 2011). miR-K10a and miR-142-3p both have two $5^{\prime}$ processing variants, which are expressed at close to a 1:1 ratio (Umbach and Cullen, 2010; Gottwein et al., 2011). The longer miR-K10a_+1_5 shares 7 mer seed homology with the shorter miR-142-3p-1_5p (Figure 3A). Because the $5^{\prime}$ nt of miR-K10a is a uridine, $7 \mathrm{mer}-1 \mathrm{~A}$ sites for miR-K10a are also $7 \mathrm{mer}$ sites for miR-K10a $+1 \_5$ and both miRNAs expressed by miR-K10a are predicted to effectively mimic miR-142-3p-1_5. In fact, only $15 \%$ of the PAR-CLIP sites predicted as targets of the two miR-K10a variants were not also predicted to be targets of miR-142-3p-1_5. miR-142-3p is expressed in all cells of the hematopoietic lineage and largely absent in non-hematopoietic cells, but very few targets and functions of miR-142-3p have been identified to date. In PEL cell lines, miR-142-3p is likely significantly more abundant than miR-K10, suggesting that these miRNAs may be redundant in $\mathrm{B}$ cells. Because miR-142-3p is absent from many other cell types, including endothelial cells, it is possible that KSHV uses miR$\mathrm{K} 10$ to introduce miR-142-3p-like functions into other cell types. Interestingly, PAR-CLIP sites assigned to miR-K10/miR-142-3p were also reported in latent viral mRNAs (Gottwein et al., 2011), including the $3^{\prime}$ UTRs of v-cyclin/LANA, the KSHV homolog of human IL-6 (v-IL-6), and latent nuclear antigen 2/viral interferon regulatory factor 3 , a protein that interferes with cellular signaling and is essential for the survival of PEL cells in culture (Wies et al., 2008). While these interactions remain to be validated at a functional level, their identification raises the possibility that KSHV uses miR-142-3p and/or miR-K10 to fine tune latent protein expression. In conclusion, while KSHV clearly encodes functional mimics of cellular miRNAs, it is also important to point out that this analogy extends only to the seed region. Like in the case of viral homologs of cellular proteins, it appears likely that viral analogs of cellular miRNAs also differ in key aspects from their cellular counterparts. Although such differences remain to be identified, these may include differences in non-seed target interactions, miRNA stability, and miRNA regulation.

\section{DO THE KSHV miRNAS REGULATE REACTIVATION?}

The establishment of latency, a highly restricted gene expression pattern that mediates the maintenance of the viral episome and minimizes immune recognition, is universal among herpesviruses and critical for their life-long persistence. Expression of herpesviral immediate early (IE) genes serves as a "switch" that triggers reactivation from latency and initiates the lytic gene expression cascade that ultimately results in expression of all viral genes and the production of progeny virus. RTA is both necessary and sufficient for the initiation of lytic KSHV replication. RTA expression is tightly regulated to respond to a variety of physiological triggers. While the default gene expression program of KSHV in culture is latency, spontaneous reactivation occurs at a low rate (typically $\sim 1-2 \%$ of the cells). Latently expressed $\alpha$-herpesviral miRNAs were found to regulate IE genes (Umbach et al., 2008; Jurak et al., 2011) and it is an attractive hypothesis that viral miRNAs in general function in this manner to stabilize latency (Murphy et al., 2008). Potentially, regulation of RTA could take place at the level of transcription, through epigenetic modification and the availability of repressors and activators of transcription, at the level of mRNA stability and translation and at the level of protein stability and activity. Thus, in order to affect RTA levels, the KSHV miRNAs may directly target RTA mRNAs or regulate cellular factors that control RTA expression and activity. Two independent studies reported that deletion of the intronic miRNAs, miR-K1-9 and miR-K11, from KSHV bac36 causes a two to fourfold increase in RTA transcript levels, a similarly modest increase in downstream viral lytic gene expression and an approximately twofold increase in virus yield from $293 \mathrm{~T}$ cells, suggesting that the intronic miRNAs modulate the latent to lytic switch in favor of latency (Lei et al., 2010; Lu et al., 2010b). This phenotype was accompanied by a modest, 1.5- to 2-fold, increase in RTA promoter reporter activity in these cells (Lei et al., 2010), suggesting that regulation is at the level of promoter activity. While Lei et al. (2010) reported that this effect could be reversed by re-expression of miR-K1, Lu et al. (2010b) observed no restoration of RTA inhibition by re-expression of miR-K1 and presented data suggesting that the expression of miR-K5, and perhaps miR-K4, was able to restore lower RTA transcript levels. Given that a similar cell type was used by both groups, it is possible that differences in the level of the re-expressed miRNAs may account for the discrepancies between these studies. In a complementary approach, Bellare and Ganem (2009) transfected antisense inhibitors against individual miRNAs into human foreskin fibroblasts (HFFs) infected with rKSHV.219, a recombinant KSHV containing an RFP reporter under control of the lytic PAN promoter (Vieira and O'Hearn, 2004). Interestingly, no effect on the spontaneous reactivation of KSHV was observed upon individual inhibition of either miR-K1, miR-K4-5p, miRK4-3p, or miR-K5, suggesting that, at least in HFF, inhibition of these miRNAs alone is not sufficient to cause an increase in spontaneous reactivation. In HFF, only the inhibition of miR-K9-5p resulted in a $\sim 2.5$-fold increase in RFP expression, likely due to a direct interaction of this miRNA with a site in the $3^{\prime}$ UTR of RTA (Bellare and Ganem, 2009), but expression of miR-K9 was reported to be unable to restore RTA inhibition to the miRNAdeleted virus in 293 cells (Lu et al., 2010b). Another study reported that overexpression of miR-K3 in the PEL cell line BC-3 results in decreased RTA mRNA expression (Lu et al., 2010a), but the contribution of endogenously expressed miR-K3 to the maintenance of latency in PEL remains unclear. The re-expression of miR-K3 was unable to restore RTA inhibition to the miRNA-deleted virus in 293 cells (Lu et al., 2010b) and its inhibition did not result in reactivation of the virus in HFF (Bellare and Ganem, 2009). In addition to the study by Bellare and Ganem (2009), several laboratories have tested RTA-3' UTR indicators for inhibition by the KSHV miRNAs, with different results (Lei et al., 2010; Lu et al., 2010b; Lin et al., 2011). Additional miRNAs that were reported to inhibit RTA indicator expression include miR-K5 and miR-K7-5p (Lu et al., 2010b; Lin et al., 2011). Lei et al. (2010), however, did not observe RTA indicator repression by any of the KSHV miRNAs. It appears that differences between studies are most likely due to the use of different approaches to miRNA expression, using mimics or from expression vectors, which may either overexpress or inadequately express $5 \mathrm{p}$ and $3 \mathrm{p}$ miRNAs. Another source of experimental discrepancies could be the folding of the $3^{\prime}$ UTRindicator transcript which depends on the exact sequence of the 
transcript. It is conceivable that unnatural $3^{\prime}$ UTR folding could result in both false positive and false negative results. While the above studies generally agree that the KSHV miRNAs modestly stabilize latency, Ziegelbauer et al. (2009) showed that the combined inhibition of miR-K5, miR-K9-3p, and miR-K10a/b in PEL and SLK cells had the opposite effect and reduced the percentage of cells undergoing spontaneous reactivation by about $40 \%$, likely through de-repression of the cellular protein bcl2-associated factor (BCLAF1). Thus, several of the miRNAs may also help tip the balance in favor of lytic reactivation. In conclusion, while much work remains to be done to establish which individual or collaborating KSHV miRNAs affect KSHV reactivation in different cell types, existing data point to a subtle function of the intronic miRNAs in favor of maintaining latency. The observed effects were modest and may present additional controls to KSHV gene expression. It is plausible that the KSHV miRNAs are under evolutionary pressure to be compatible with the latent and lytic replication modes, and even possible that they have evolved to avoid strong interactions with RTA and other lytic mRNAs.

\section{TRANSCRIPTIONAL AND EPIGENETIC REPROGRAMMING}

While direct recognition by miRNAs often results in only subtle changes of target expression, indirect functional consequences can be more dramatic if regulated mRNAs encode transcription factors and other regulators of gene expression. Transcriptional regulation was the most highly enriched function among candidate targets of the KSHV miRNAs (Gottwein et al., 2011), and several transcription factors have already been validated as targets of KSHV miRNAs. KSHV-infected spindle cells are the predominant cell type in advanced KS lesion. Spindle cells are poorly differentiated with expression of lymphatic and blood vessel endothelial cell (LEC and BEC) as well as mesenchymal markers. This transcriptional reprogramming is recapitulated upon infection of LECs by KSHV in vitro (Boshoff et al., 1995; Hong et al., 2004; Wang et al., 2004; Cheng et al., 2011; Gasperini et al., 2012). Several KSHV miRNAs contribute to the transcriptional reprogramming of LEC by KSHV through targeting of the transcription factor cMaf (MAF; Hansen et al., 2010). Large Maf transcription factors, including c-Maf, can act as activators or repressors and regulate the terminal differentiation of a number of tissues. c-Maf is among the proteins that are specifically induced in LEC by the transcriptional master regulator of lymphatic endothelial differentiation PROX1 (Petrova et al., 2002; Hong et al., 2004). c-Maf was downregulated in LEC stably expressing the intronic KSHV miRNAs and found to be directly repressed by KSHV miR-K6 and miR-K11. Downregulation of c-Maf following the expression of the intronic miRNAs or by RNAi induced the expression of BEC marker genes in LEC, suggesting that c-Maf is important to maintain LEC differentiation. Thus, regulation of MAF by the KSHV miRNAs at least partially accounts for the transcriptional reprogramming observed upon KSHV infection of LEC. Other examples of transcription factors targeted by KSHV miRNAs include the transcriptional repressors RBL2 (retinoblastoma-like 2), BACH1 (BTB and CNC homology 1, basic leucine zipper transcription factor 1), and BCLAF1 (discussed above and below). RBL2 is downregulated by miR-K4-5p, potentially resulting in increased expression of DNA methyl transferases DNMT1, 3a, and 3b (Lu et al., 2010b). Consistent with a function of the KSHV miRNAs in the epigenetic modification of the KSHV and cellular genomes, deletion of the intronic miRNAs resulted in an overall reduction of $\mathrm{CpG}$ methylation of the viral and cellular genomes. $\mathrm{BACH} 1$ is among the most strongly regulated targets of the KSHV miRNAs validated to date (Gottwein et al., 2007; Skalsky et al., 2007). The best characterized target of BACH1-mediated repression is hemeoxygenase 1 (HO-1, HMOX1), the limiting enzyme in heme catabolism, which is strongly upregulated in KS (McAllister et al., 2004). More recently, Qin et al. (2010a) showed that BACH1 also represses SLC7A11 (solute carrier family 7, $\mathrm{xCT}$ ), a transporter which imports cystine required for the synthesis of glutathione (GSH), a critical antioxidant that protects cells from reactive oxygen species (ROS). Consequently, the resulting upregulation of SLC7A11 protects infected cells from oxidative stress. BACH1-ChIP-seq in 293 cells (Warnatz et al., 2011), which express endogenous BACH1, confirmed HO-1 and SLC7A11 as BACH1 target genes and identified additional targets of $\mathrm{BACH} 1$, including additional factors involved in redox and transport processes, and those involved in cell cycle and apoptosis. Thus, several transcription factors have already been shown to be important targets of the KSHV miRNAs and many more are likely candidates, suggesting that transcriptional reprogramming is an important function of the KSHV miRNAs.

\section{EVASION FROM CELL CYCLE ARREST AND CELL DEATH}

The deregulation of the cell cycle, survival signaling, and cell death pathways contributes to the oncogenic properties of KSHV and other oncogenic viruses. Not surprisingly, several of the already validated targets of the KSHV miRNAs are predicted to facilitate viral escape from cell cycle arrest and apoptosis. KSHV miR-K1 targets p21, a cyclin-dependent kinase inhibitor (CDKI) induced by several growth inhibitory signals, and thereby attenuates p21mediated cell cycle arrest (Gottwein and Cullen, 2010). More recently, several other miRNAs were reported to also bind the p21 $3^{\prime}$ UTR and the $3^{\prime}$ UTR of a second CDKI, p27, but the functional impact of these interactions in the context of KSHV infection remains to be established (Gottwein et al., 2011). As discussed above, the downregulation of BACH1 by miR-K11 promotes cellular survival under oxidative stress, through de-repression of xCT (Qin et al., 2010a). KSHV miR-K10 was shown to allow the escape from TNFSF12/TWEAK-induced apoptosis by repression of TNRSFR12A/TWEAKR, its receptor (Abend et al., 2010), and miRNAs miR-K1, miR-K3, and miR-K4-3p target effector caspase 3 (CASP3; Suffert et al., 2011). The co-expression of the intronic KSHV miRNAs inhibited apoptosis following treatment with staurosporine, while the joint inhibition of miR-K1, miR$\mathrm{K} 3$, and miR-K4-3p in KSHV-infected E6/E7-immortalized LEC enhanced etoposide-induced apoptosis by about twofold (Suffert et al., 2011). Attenuation of staurosporine-induced apoptosis was also observed in DG-75 cells in the absence of caspase 3 activation, which is defective in these cells, suggesting that caspase 3 is unlikely to be the functionally relevant target in DG-75 cells. An important remaining question is whether an approximately twofold reduction of procaspase 3 expression can indeed protect cells from cell death or even delay its progression, given that caspase 3 is an effector rather than an inducer of apoptosis and may 
not be limiting to the progression of apoptosis. TGF- $\beta$ is a multifunctional cytokine that induces cell cycle arrest and apoptosis in B cells. Importantly, PEL cell lines are generally resistant to the effects of TGF- $\beta$, at least in part due to inhibition of TGF- $\beta$ receptor 2 subunit expression by LANA (Di Bartolo et al., 2008). The stable expression of the intronic KSHV miRNAs in 293T resulted in strongly reduced activity of a SMAD-responsive element reporter (Samols et al., 2007), suggesting that the KSHV miRNAs may also contribute to the inhibition of TGF- $\beta$ signaling by KSHV or at least serve as an additional safeguard. The mechanism underlying this observation was not addressed, but, more recently it was shown that miR-K11, like its analog miR-155 (Yin et al., 2008, 2010; Rai et al., 2010), antagonizes SMAD5 and this interaction likely contributes to the observed resistance to TGF-beta induced growth inhibition and apoptosis in PEL (Liu et al., 2012). Other candidate targets with known roles in the regulation of cell cycle and cell death have been validated (Dölken et al., 2010; Gottwein et al., 2011), but are of unknown relevance in the context of KSHV infection.

\section{OTHER FUNCTIONS OF THE KSHV miRNAs}

Immune evasion is critical for the life-long persistence of herpesviral infection and herpesviruses employ multiple mechanisms to ensure the escape of infected cells from both the innate and the adaptive arms of the immune system. Latency and the widespread use of viral miRNA expression during latency themselves can be viewed as mechanisms of passive immune evasion. One important target with a role in evasion from natural killer (NK) cells is the miR-K7 target MICB (Nachmani et al., 2009), whose surface expression triggers recognition by the NK cell activating receptor NKG2D and subsequent killing by NK cells. MICB is also repressed by the miRNAs of other herpesviruses, including EBV miR-Bart2-5p and HCMV miR-UL112, through different sites (Stern-Ginossar et al., 2007; Nachmani et al., 2009), which further underscores the likely importance of this target in herpesviral infection. A final important emerging concept is the regulation of cellular signaling by the KSHV miRNAs and many of the validated targets discussed above participate in one or more signaling cascades (Table $\mathbf{1}$ ). Given the critical role cytokines play in the pathogenesis of KS, deregulation of cytokine expression by KSHV miRNAs is perhaps expected. Abend et al. (2010)

\section{REFERENCES}

Abend, J. R., Uldrick, T., and Ziegelbauer, J. M. (2010). Regulation of tumor necrosis factor-like weak inducer of apoptosis receptor protein (TWEAKR) expression by Kaposi's sarcoma-associated herpesvirus microRNA prevents TWEAK-induced apoptosis and inflammatory cytokine expression. J. Virol. 84, 12139-12151.

AuCoin, D. P., Colletti, K. S., Xu, Y., Cei, S. A., and Pari, G. S. (2002). Kaposi's sarcoma-associated herpesvirus (human herpesvirus 8) contains two functional lytic origins of DNA replication. J. Virol. 76, 7890-7896.

demonstrated that the ectopic expression of miR-K10a in human umbilical vein endothelial cells (HUVECs) inhibited the TWEAKinduced expression of the proinflammatory cytokines IL-8 and MCP-1 approximately two to threefold. Another report has implicated several KSHV miRNAs in the induction of IL-6 and IL-10 secretion by murine and human myeloid-derived cells, potentially through downregulation of the C/EBP $\beta$ LIP isoform (Qin et al., 2010b). However, this effect is unlikely to be entirely due to a direct interaction of the proposed KSHV miRNAs with the $\mathrm{C} / \mathrm{EBP} \beta$ mRNA, because $\mathrm{C} / \mathrm{EBP} \beta$ isoforms other than LIP, which are expressed from the same mRNA through differential translational initiation (Calkhoven et al., 2000), were not affected. In addition, the murine and human $\mathrm{C} / \mathrm{EBP} \beta$ mRNAs lack canonical miRNA binding sites for miR-K3 and miR-K7. C/EBP $\beta$, however, is a well validated target of cellular miR-155 (O'Connell et al., 2008; Costinean et al., 2009) and is also regulated by its analog miR-K11 (Boss et al., 2011). Lei et al. (2010) demonstrated that KSHV miR-K1 inhibits $\mathrm{I} \kappa \mathrm{B} \alpha$ expression and consequently activates NFKB signaling, which may help stabilize KSHV latency. Finally, PAR-CLIP data from PEL cells identified numerous candidates for direct targets of the KSHV miRNAs with roles in signaling (Gottwein et al., 2011).

\section{CONCLUSION AND OUTLOOK}

An important open question in the miRNA field is whether miRNAs have a small number of critical targets or whether many, possibly hundreds of interactions of each individual miRNA have functional importance. The KSHV miRNAs have undoubtedly evolved a very complex set of regulatory relationships through viral analogs of cellular miRNAs and also novel miRNA sequences. Future functional experiments will have to clarify how many and which of these interactions are important for the KSVH life cycle and pathogenesis. Particularly relevant will be to elucidate functions of the KSHV miRNAs that contribute to the development of KSHV-induced disease, which could become targets for therapeutic intervention.

\section{ACKNOWLEDGMENTS}

Work in the Gottwein laboratory is supported by the NCI (R00CA137860). Eva Gottwein would like to thank Eleonora Forte and Jonathan Leis for comments on the manuscript.

sarcoma-associated herpesvirus infects endothelial and spindle cells. Nat. Med. 1, 1274-1278.

Boss, I. W., Nadeau, P. E., Abbott, J. R., Yang, Y., Mergia, A., and Renne, R. (2011). A Kaposi's sarcoma-associated herpesvirusencoded ortholog of microRNA miR-155 induces human splenic B-cell expansion in NOD/LtSz-scid IL2Rgammanull mice. J. Virol. 85, 9877-9886.

Cai, X., and Cullen, B. R. (2006). Transcriptional origin of Kaposi's sarcoma-associated herpesvirus microRNAs. J. Virol. 80, 2234-2242. Cai, X., Lu, S., Zhang, Z., Gonzalez, C. M., Damania, B., and Cullen, B. R.
(2005). Kaposi's sarcoma-associated herpesvirus expresses an array of viral microRNAs in latently infected cells. Proc. Natl. Acad. Sci. U.S.A. 102, 5570-5575.

Cai, X., Schafer, A., Lu, S., Bilello, J. P., Desrosiers, R. C., Edwards, R., Raab-Traub, N., and Cullen, B. R. (2006). Epstein-Barr virus microRNAs are evolutionarily conserved and differentially expressed. PLoS Pathog. 2, e23. doi:10.1371/journal.ppat.0020023

Calkhoven, C. F., Muller, C., and Leutz, A. (2000). Translational control of C/EBPalpha and C/EBPbeta isoform expression. Genes Dev. 14, 1920-1932. 
Cesarman, E., Chang, Y., Moore, P. S., Said, J. W., and Knowles, D. M. (1995). Kaposi's sarcoma-associated herpesvirus-like DNA sequences in AIDS-related body-cavity-based lymphomas. N. Engl. J. Med. 332, 1186-1191.

Chandriani, S., Xu, Y., and Ganem, D. (2010). The lytic transcriptome of Kaposi's sarcoma-associated herpesvirus reveals extensive transcription of noncoding regions, including regions antisense to important genes. J. Virol. 84, 7934-7942.

Cheng, F., Pekkonen, P., Laurinavicius, S., Sugiyama, N., Henderson, S., Gunther, T., Rantanen, V., Kaivanto, E., Aavikko, M., Sarek, G., Hautaniemi, S., Biberfeld, P., Aaltonen, L., Grundhoff, A., Boshoff, C., Alitalo, K., Lehti, K., and Ojala, P. M. (2011). KSHV-initiated notch activation leads to membrane-type-1 matrix metalloproteinase-dependent lymphatic endothelial-to-mesenchymal transition. Cell Host Microbe 10, 577-590.

Chiang, H. R., Schoenfeld, L. W., Ruby, J. G., Auyeung, V. C., Spies, N., Baek, D., Johnston, W. K., Russ, C., Luo, S., Babiarz, J. E., Blelloch, R., Schroth, G. P., Nusbaum, C., and Bartel, D. P. (2010). Mammalian microRNAs: experimental evaluation of novel and previously annotated genes. Genes Dev. 24, 992-1009.

Costinean, S., Sandhu, S. K., Pedersen, I. M., Tili, E., Trotta, R., Perrotti, D., Ciarlariello, D., Neviani, P., Harb, J., Kauffman, L. R., Shidham, A., and Croce, C. M. (2009). Src homology 2 domain-containing inositol-5phosphatase and CCAAT enhancerbinding protein beta are targeted by miR-155 in B cells of EmicroMiR-155 transgenic mice. Blood 114, 1374-1382.

Costinean, S., Zanesi, N., Pekarsky, Y., Tili, E., Volinia, S., Heerema, N., and Croce, C. M. (2006). Pre-B cell proliferation and lymphoblastic leukemia/high-grade lymphoma in $\mathrm{E}(\mathrm{mu})$-miR155 transgenic mice. Proc. Natl. Acad. Sci. U.S.A. 103, 7024-7029.

Cullen, B. R. (2006). Viruses and microRNAs. Nat. Genet. 38(Suppl.), S25-S30.

Cullen, B. R. (2011). Viruses and microRNAs: RISCy interactions with serious consequences. Genes Dev. 25, 1881-1894.

Di Bartolo, D. L., Cannon, M., Liu, Y. F., Renne, R., Chadburn, A., Boshoff, C., and Cesarman, E. (2008). KSHV LANA inhibits TGF-beta signaling through epigenetic silencing of the TGF-beta type II receptor. Blood 111, 4731-4740.

Dölken, L., Malterer, G., Erhard, F., Kothe, S., Friedel, C. C., Suffert, G., Marcinowski, L., Motsch, N., Barth, S., Beitzinger, M., Lieber, D., Bailer, S. M., Hoffmann, R., Ruzsics, Z., Kremmer, E., Pfeffer, S., Zimmer, R., Koszinowski, U. H., Grasser, F., Meister, G., and Haas, J. (2010). Systematic analysis of viral and cellular microRNA targets in cells latently infected with human gamma-herpesviruses by RISC immunoprecipitation assay. Cell Host Microbe 7, 324-334.

Gandy, S. Z., Linnstaedt, S. D., Muralidhar, S., Cashman, K. A., Rosenthal, L. J., and Casey, J. L. (2007). RNA editing of the human herpesvirus 8 kaposin transcript eliminates its transforming activity and is induced during lytic replication. J. Virol. 81, 13544-13551.

Gasperini, P., Espigol-Frigole, G., Mccormick, P. J., Salvucci, O., Maric, D., Uldrick, T. S., Polizzotto, M. N., Yarchoan, R., and Tosato, G. (2012). Kaposi sarcoma herpesvirus promotes endothelialto-mesenchymal transition through Notch-dependent signaling. Cancer Res. 72, 1157-1169.

Gottwein, E., Cai, X., and Cullen, B. R. (2006). A novel assay for viral microRNA function identifies a single nucleotide polymorphism that affects Drosha processing. J. Virol. 80, 5321-5326.

Gottwein, E., Corcoran, D. L., Mukherjee, N., Skalsky, R. L., Hafner, M., Nusbaum, J. D., Shamulailatpam, P., Love, C. L., Dave, S. S., Tuschl, T., Ohler, U., and Cullen, B. R. (2011). Viral microrna targetome of KSHV-infected primary effusion lymphoma cell lines. Cell Host Microbe 10, 515-526.

Gottwein, E., and Cullen, B. R. (2010). A human herpesvirus microRNA inhibits p21 expression and attenuates p21-mediated cell cycle arrest. J. Virol. 84, 5229-5237.

Gottwein, E., Mukherjee, N., Sachse, C., Frenzel, C., Majoros, W. H., Chi, J. T., Braich, R., Manoharan, M., Soutschek, J., Ohler, U., and Cullen, B. R. (2007). A viral microRNA functions as an orthologue of cellular miR-155. Nature 450, 1096-1099.

Grey, F., Tirabassi, R., Meyers, H., Wu, G., Mcweeney, S., Hook, L., and Nelson, J. A. (2010). A viral microRNA down-regulates multiple cell cycle genes through mRNA 5'UTRs. PLoS Pathog. 6, el000967. doi:10.1371/journal.ppat.1000967
Grundhoff, A., and Sullivan, C. S. (2011). Virus-encoded microRNAs. Virology 411, 325-343.

Grundhoff, A., Sullivan, C. S., and Ganem, D. (2006). A combined computational and microarraybased approach identifies novel microRNAs encoded by human gamma-herpesviruses. RNA 12, 733-750.

Hafner, M., Landthaler, M., Burger, L. Khorshid, M., Hausser, J., Berninger, P., Rothballer, A., Ascano, M. Jr., Jungkamp, A. C., Munschauer, M. Ulrich, A., Wardle, G. S., Dewell, S., Zavolan, M., and Tuschl, T. (2010). Transcriptome-wide identification of RNA-binding protein and microRNA target sites by PAR-CLIP. Cell 141, 129-141.

Hansen, A., Henderson, S., Lagos, D. Nikitenko, L., Coulter, E., Roberts, S. Gratrix, F., Plaisance, K., Renne, R., Bower, M., Kellam, P., and Boshoff, C. (2010). KSHV-encoded miRNAs target MAF to induce endothelial cell reprogramming. Genes Dev. 24, 195-205.

Hong, Y. K., Foreman, K., Shin, J. W. Hirakawa, S., Curry, C. L., Sage, D. R., Libermann, T., Dezube, B. J., Fingeroth, J. D., and Detmar, M. (2004). Lymphatic reprogramming of blood vascular endothelium by Kaposi sarcoma-associated herpesvirus. Nat. Genet. 36, 683-685.

Huntzinger, E., and Izaurralde, E. (2011). Gene silencing by microRNAs: contributions of translational repression and mRNA decay. Nat. Rev. Genet. 12, 99-110.

Hutvagner, G., and Zamore, P. D (2002). A microRNA in a multipleturnover RNAi enzyme complex. Science 297, 2056-2060.

Jurak, I., Griffiths, A., and Coen, D. M. (2011). Mammalian alphaherpesvirus miRNAs. Biochim. Biophys. Acta 1809, 641-653.

Kim, V. N., Han, J., and Siomi, M. C. (2009). Biogenesis of small RNAs in animals. Nat. Rev. Mol. Cell Biol. 10, 126-139.

Lagos-Quintana, M., Rauhut, R., Lendeckel, W., and Tuschl, T. (2001). Identification of novel genes coding for small expressed RNAs. Science 294, 853-858.

Langenberger, D., Bermudez-Santana, C., Hertel, J., Hoffmann, S., Khaitovich, P., and Stadler, P. F. (2009). Evidence for human microRNA-offset RNAs in small RNA sequencing data. Bioinformatics 25, 2298-2301.

Lau, N. C., Lim, L. P., Weinstein, E. G., and Bartel, D. P. (2001).
An abundant class of tiny RNAs with probable regulatory roles in Caenorhabditis elegans. Science 294, 858-862.

Lee, R. C., and Ambros, V. (2001). An extensive class of small RNAs in Caenorhabditis elegans. Science 294, 862-864.

Lee, R. C., Feinbaum, R. L., and Ambros, V. (1993). The C. elegans heterochronic gene lin-4 encodes small RNAs with antisense complementarity to lin-14. Cell 75, 843-854.

Lei, X., Bai, Z., Ye, F., Xie, J., Kim, C. G., Huang, Y., and Gao, S. J. (2010). Regulation of NF-kappaB inhibitor IkappaBalpha and viral replication by a KSHV microRNA. Nat. Cell Biol. 12, 193-199.

Liang, D., Gao, Y., Lin, X., He, Z., Zhao, Q., Deng, Q., and Lan, K. (2011). A human herpesvirus miRNA attenuates interferon signaling and contributes to maintenance of viral latency by targeting IKKepsilon. Cell Res. 21, 793-806.

Lin, C. L., Li, H., Wang, Y., Zhu, F. X., Kudchodkar, S., and Yuan, Y. (2003). Kaposi's sarcomaassociated herpesvirus lytic origin (ori-Lyt)-dependent DNA replication: identification of the ori-Lyt and association of $\mathrm{K} 8$ bZip protein with the origin. J. Virol. 77, 5578-5588.

Lin, H. R., and Ganem, D. (2011). Viral microRNA target allows insight into the role of translation in governing microRNA target accessibility. Proc. Natl. Acad. Sci. U.S.A. 108, 5148-5153.

Lin, X., Liang, D., He, Z., Deng, Q., Robertson, E. S., and Lan, K. (2011). miR-K12-7-5p encoded by Kaposi's sarcoma-associated herpesvirus stabilizes the latent state by targeting viral ORF50/RTA. PLoS ONE 6, e16224. doi:10.1371/journal.pone.0016224

Lin, Y. T., Kincaid, R. P., Arasappan, D., Dowd, S. E., Hunicke-Smith, S. P., and Sullivan, C. S. (2010). Small RNA profiling reveals antisense transcription throughout the KSHV genome and novel small RNAs. RNA 16, 1540-1558.

Lin, Y. T., and Sullivan, C. S. (2011). Expanding the role of Drosha to the regulation of viral gene expression. Proc. Natl. Acad. Sci. U.S.A. 108, 11229-11234.

Linnstaedt, S. D., Gottwein, E., Skalsky, R. L., Luftig, M. A., and Cullen, B. R. (2010). Virally induced cellular microRNA miR-155 plays a key role in B-cell immortalization by Epstein-Barr virus. J. Virol. 84, 11670-11678. 
Liu, Y., Sun, R., Lin, X., Liang, D., Deng, Q., and Lan, K. (2012). KSHV-encoded miR-K12-11 attenuates transforming growth factor beta signaling through suppression of SMAD5. J. Virol. 86, 1372-1381.

Lu, C. C., Li, Z., Chu, C. Y., Feng, J., Sun, R., and Rana, T. M. (2010a). MicroRNAs encoded by Kaposi's sarcomaassociated herpesvirus regulate viral life cycle. EMBO Rep. 11, 784-790.

Lu, F., Stedman, W., Yousef, M., Renne, R., and Lieberman, P. M. (2010b). Epigenetic regulation of Kaposi's sarcoma-associated herpesvirus latency by virus-encoded microRNAs that target Rta and the cellular Rbl2-DNMT pathway. $J$. Virol. 84, 2697-2706.

Lu, F., Weidmer, A., Liu, C. G., Volinia, S., Croce, C. M., and Lieberman, P. M. (2008). Epstein-Barr virus-induced miR-155 attenuates NF-kappaB signaling and stabilizes latent virus persistence. J. Virol. 82, 10436-10443.

Marshall, V., Martro, E., Labo, N., Ray, A., Wang, D., Mbisa, G., Bagni, R. K., Volfovsky, N., Casabona, J., and Whitby, D. (2010). Kaposi sarcoma (KS)-associated herpesvirus microRNA sequence analysis and KS risk in a European AIDS-KS case control study. J. Infect. Dis. 202, 1126-1135.

Marshall, V., Parks, T., Bagni, R., Wang, C. D., Samols, M. A., Hu, J., Wyvil, K. M., Aleman, K., Little, R. F., Yarchoan, R., Renne, R., and Whitby, D. (2007). Conservation of virally encoded microRNAs in Kaposi sarcoma - associated herpesvirus in primary effusion lymphoma cell lines and in patients with Kaposi sarcoma or multicentric Castleman disease. J. Infect. Dis. 195, 645-659.

McAllister, S. C., Hansen, S. G., Ruhl, R. A., Raggo, C. M., Defilippis, V. R., Greenspan, D., Fruh, K., and Moses, A. V. (2004). Kaposi sarcoma-associated herpesvirus (KSHV) induces heme oxygenase-1 expression and activity in KSHVinfected endothelial cells. Blood 103, 3465-3473.

Murphy, E., Vanicek, J., Robins, H., Shenk, T., and Levine, A. J. (2008). Suppression of immediate-early viral gene expression by herpesviruscoded microRNAs: implications for latency. Proc. Natl. Acad. Sci. U.S.A. 105, 5453-5458.

Nachmani, D., Stern-Ginossar, N., Sarid, R., and Mandelboim, O. (2009). Diverse herpesvirus microRNAs target the stress-induced immune ligand MICB to escape recognition by natural killer cells. Cell Host Microbe 5, 376-385.

O'Connell, R. M., Rao, D. S., Chaudhuri, A. A., Boldin, M. P., Taganov, K. D., Nicoll, J., Paquette, R. L., and Baltimore, D. (2008). Sustained expression of microRNA-155 in hematopoietic stem cells causes a myeloproliferative disorder. J. Exp. Med. 205, 585-594.

O’Hara, A. J., Chugh, P., Wang, L., Netto, E. M., Luz, E., Harrington, W. J., Dezube, B. J., Damania, B., and Dittmer, D. P. (2009). Pre-micro RNA signatures delineate stages of endothelial cell transformation in Kaposi sarcoma. PLoS Pathog. 5, e1000389. doi:10.1371/journal.ppat.1000389

Pasquinelli, A. E., Reinhart, B. J., Slack, F., Martindale, M. Q., Kuroda, M. I., Maller, B., Hayward, D. C., Ball, E. E., Degnan, B., Muller, P., Spring, J., Srinivasan, A., Fishman, M., Finnerty, J., Corbo, J., Levine, M., Leahy, P., Davidson, E., and Ruvkun, G. (2000). Conservation of the sequence and temporal expression of let-7 heterochronic regulatory RNA. Nature 408, 86-89.

Petrova, T. V., Makinen, T., Makela, T. P., Saarela, J., Virtanen, I., Ferrell, R. E., Finegold, D. N., Kerjaschki, D., YlaHerttuala, S., and Alitalo, K. (2002). Lymphatic endothelial reprogramming of vascular endothelial cells by the Prox-1 homeobox transcription factor. EMBO J. 21, 4593-4599.

Pfeffer, S., Sewer, A., Lagos-Quintana, M., Sheridan, R., Sander, C., Grasser, F. A., Van Dyk, L. F., Ho, C. K., Shuman, S., Chien, M., Russo, J. J., Ju, J., Randall, G., Lindenbach, B. D. Rice, C. M., Simon, V., Ho, D. D., Zavolan, M., and Tuschl, T. (2005). Identification of microRNAs of the herpesvirus family. Nat. Methods 2, 269-276.

Pfeffer, S., Zavolan, M., Grasser, F. A., Chien, M., Russo, J. J., Ju, J., John, B., Enright, A. J., Marks, D., Sander, C., and Tuschl, T. (2004). Identification of virus-encoded microRNAs. Science 304, 734-736.

Qin, Z., Freitas, E., Sullivan, R., Mohan, S., Bacelieri, R., Branch, D., Romano, M., Kearney, P., Oates, J., Plaisance, K., Renne, R., Kaleeba, J., and Parsons, C. (2010a). Upregulation of xCT by KSHV-encoded microRNAs facilitates KSHV dissemination and persistence in an environment of oxidative stress. PLoS Pathog. 6, e1000742. doi:10.1371/journal.ppat.1000742

Qin, Z., Kearney, P., Plaisance, K., and Parsons, C. H. (2010b).
Pivotal advance: Kaposi's sarcoma-associated herpesvirus (KSHV)-encoded microRNA specifically induce IL-6 and IL-10 secretion by macrophages and monocytes. J. Leukoc. Biol. 87, 25-34.

Rai, D., Kim, S. W., Mckeller, M. R., Dahia, P. L., and Aguiar, R. C. (2010). Targeting of SMAD5 links microRNA-155 to the TGFbeta pathway and lymphomagenesis. Proc. Natl. Acad. Sci. U.S.A. 107, 3111-3116.

Reinhart, B. J., Slack, F. J., Basson, M., Pasquinelli, A. E., Bettinger, J. C., Rougvie, A. E., Horvitz, H. R., and Ruvkun, G. (2000). The 21-nucleotide let-7 RNA regulates developmental timing in Caenorhabditis elegans. Nature 403 901-906.

Samols, M. A., Hu, J., Skalsky, R. L., and Renne, R. (2005). Cloning and identification of a microRNA cluster within the latency-associated region of Kaposi's sarcomaassociated herpesvirus. J. Virol. 79, 9301-9305.

Samols, M. A., Skalsky, R. L., Maldonado, A. M., Riva, A., Lopez, M. C., Baker, H. V., and Renne, R. (2007). Identification of cellular genes targeted by KSHV-encoded microRNAs. PLoS Pathog. 3, e65. doi:10.1371/journal.ppat.0030065

Selbach, M., Schwanhausser, B., Thierfelder, N., Fang, Z., Khanin, R., and Rajewsky, N. (2008). Widespread changes in protein synthesis induced by microRNAs. Nature 455 58-63.

Shi, W., Hendrix, D., Levine, M., and Haley, B. (2009). A distinct class of small RNAs arises from premiRNA-proximal regions in a simple chordate. Nat. Struct. Mol. Biol. 16, 183-189.

Shin, C., Nam, J. W., Farh, K. K., Chiang, H. R., Shkumatava, A., and Bartel, D. P. (2010). Expanding the microRNA targeting code: functional sites with centered pairing. Mol. Cell 38, 789-802.

Skalsky, R. L., and Cullen, B. R. (2011). Reduced expression of brain-enriched microRNAs in glioblastomas permits targeted regulation of a cell death gene. PLoS ONE 6, e24248. doi:10.1371/journal.pone.0024248

Skalsky, R. L., Samols, M. A., Plaisance, K. B., Boss, I. W., Riva, A., Lopez, M. C., Baker, H. V., and Renne, R. (2007). Kaposi's sarcomaassociated herpesvirus encodes an ortholog of miR-155. J. Virol. 81, 12836-12845.
Stern-Ginossar, N., Elefant, N., Zimmermann, A., Wolf, D. G., Saleh, N., Biton, M., Horwitz, E., Prokocimer, Z., Prichard, M., Hahn, G., Goldman-Wohl, D., Greenfield, C., Yagel, S., Hengel, H., Altuvia, Y., Margalit, H., and Mandelboim, O. (2007). Host immune system gene targeting by a viral miRNA. Science 317, 376-381.

Suffert, G., Malterer, G., Hausser, J., Viiliainen, J., Fender, A., Contrant, M., Ivacevic, T., Benes, V. Gros, F., Voinnet, O., Zavolan, M., Ojala, P. M., Haas, J. G., and Pfeffer, S. (2011). Kaposi's sarcoma herpesvirus microRNAs target caspase 3 and regulate apoptosis. PLoS Pathog. 7, e1002405. doi:10.1371/journal.ppat.1002405

Umbach, J. L., and Cullen, B. R. (2010). In-depth analysis of Kaposi's sarcoma-associated herpesvirus microRNA expression provides insights into the mammalian microRNA-processing machinery. $J$. Virol. 84, 695-703.

Umbach, J. L., Kramer, M. F., Jurak, I., Karnowski, H. W., Coen, D. M., and Cullen, B. R. (2008). MicroRNAs expressed by herpes simplex virus 1 during latent infection regulate viral mRNAs. Nature 454, 780-783.

Umbach, J. L., Strelow, L. I., Wong, S. W., and Cullen, B. R. (2010). Analysis of rhesus rhadinovirus microRNAs expressed in virusinduced tumors from infected rhesus macaques. Virology 405, 592-599.

Vieira, J., and O'Hearn, P. M. (2004). Use of the red fluorescent protein as a marker of Kaposi's sarcoma-associated herpesvirus lytic gene expression. Virology 325, 225-240.

Walz, N., Christalla, T., Tessmer, U., and Grundhoff, A. (2010). A global analysis of evolutionary conservation among known and predicted gammaherpesvirus microRNAs. $J$. Virol. 84, 716-728.

Wang, H. W., Trotter, M. W., Lagos, D., Bourboulia, D., Henderson, S., Makinen, T., Elliman, S., Flanagan, A. M., Alitalo, K., and Boshoff, C. (2004). Kaposi sarcoma herpesvirus-induced cellular reprogramming contributes to the lymphatic endothelial gene expression in Kaposi sarcoma. Nat. Genet. 36, 687-693.

Warnatz, H. J., Schmidt, D., Manke, T., Piccini, I., Sultan, M., Borodina, T., Balzereit, D., Wruck, W., Soldatov, A., Vingron, M., Lehrach, H., and Yaspo, M. L. (2011). The BTB and CNC homology 1 (BACH1) target 
genes are involved in the oxidative stress response and in control of the cell cycle. J. Biol. Chem. 286, 23521-23532.

Wies, E., Mori, Y., Hahn, A., Kremmer, E., Sturzl, M., Fleckenstein, B., and Neipel, F. (2008). The viral interferon-regulatory factor3 is required for the survival of KSHV-infected primary effusion lymphoma cells. Blood 111, 320-327.

$\mathrm{Xu}, \mathrm{Y} .$, Rodriguez-Huete, A., and Pari, G. S. (2006). Evaluation of the lytic origins of replication of Kaposi's sarcoma-associated virus/human herpesvirus 8 in the context of the viral genome. J. Virol. 80, 9905-9909.
Yin, Q., Mcbride, J., Fewell, C., Lacey, M., Wang, X., Lin, Z., Cameron, J., and Flemington, E. K. (2008). MicroRNA-155 is an Epstein-Barr virus-induced gene that modulates Epstein-Barr virus-regulated gene expression pathways. J. Virol. 82, 5295-5306.

Yin, Q., Wang, X., Fewell, C., Cameron, J., Zhu, H., Baddoo, M., Lin, Z., and Flemington, E. K. (2010). MicroRNA miR-155 inhibits bone morphogenetic protein (BMP) signaling and BMP-mediated EpsteinBarr virus reactivation. J. Virol. 84, 6318-6327.

Zhao, Y., Xu, H., Yao, Y., Smith, L. P., Kgosana, L., Green, J., Petherbridge, L., Baigent, S. J., and Nair, V. (2011).
Critical role of the virus-encoded microRNA-155 ortholog in the induction of Marek's disease lymphomas. PLoS Pathog. 7, e1001305. doi:10.1371/journal.ppat.1001305

Ziegelbauer, J. M., Sullivan, C. S., and Ganem, D. (2009). Tandem array-based expression screens identify host mRNA targets of virusencoded microRNAs. Nat. Genet. 41 , 130-134.

Conflict of Interest Statement: The author declares that the research was conducted in the absence of any commercial or financial relationships that could be construed as a potential conflict of interest.
Received: 30 January 2012; paper pending published: 17 February 2012; accepted: 13 April 2012; published online: 03 May 2012.

Citation: Gottwein E (2012) Kaposi's sarcoma-associated herpesvirus microR NAs. Front. Microbio. 3:165. doi: 10.3389/fmicb.2012.00165

This article was submitted to Frontiers in Virology, a specialty of Frontiers in Microbiology.

Copyright (c) 2012 Gottwein. This is an open-access article distributed under the terms of the Creative Commons Attribution Non Commercial License, which permits non-commercial use, distribution, and reproduction in other forums, provided the original authors and source are credited. 